\title{
Site of upper airway obstruction in preterm infants with problematical apnoea
}

Department of University Hospital, Nottingham Nigel $R$ Ruggins A D Milner Correspondence to: Dr Nigel 'R Ruggins, Children's Respiratory Unit, University Hospital, Queen's Medical Centre, Nottingham NG7 2UH.

Accepted 15 January 1991

\author{
Nigel R Ruggins, A D Milner
}

\begin{abstract}
Airway obstruction is an important factor predisposing to the development of apnoea in preterm infants. An ultrafine fibreoptic scope was used to examine the upper airway of 12 preterm infants with recurrent problematical apnoea. Continuous visualisation of the airway was possible and recorded on to videotape together with simultaneous cardiorespiratory monitoring to detect apnoeic episodes in 12 infants studied. In seven infants obstruction was observed at laryngeal level with the arytenoid masses and aryepiglottic folds closing across the vocal cords. This was not only observed in obstructive apnoea but also during mixed apnoea in periods where no respiratory effort was detectable and again in apparently pure central apnoea. The findings confirm the larynx as an anatomical site of upper airway obstruction. They agree with other indirect methods of assessing airway patency that obstruction has an important role in the pathophysiology of apnoea of prematurity.
\end{abstract}

It is becoming recognised that obstruction of the upper airway is a common feature in all types of apnoea of prematurity and not limited to the relatively rare obstructive apnoea attacks. Altogether $69.5 \%$ of preterm infants with apnoea have periods of airway obstruction when episodes of obstructive and mixed apnoeas are taken into account. ${ }^{1}$ However this is clearly an underestimate if obstruction is only defined in terms of continued respiratory effort in the absence of airflow at the nose or mouth. Yet it is clear that an external obstruction will often cause preterm infants to become apnoeic even when there are no respiratory efforts against the obstruction. ${ }^{2}$ Thus obstruction of the airway can result in a 'central' apnoea as defined by standard cardiorespiratory monitors, with absent airflow and absent respiratory efforts. Clearly an indicator of airway patency would be more useful in defining the role of airway obstruction in apnoea. One approach is to measure tidal flow using a face mask system incorporating a pneumotachograph and sensitive pressure transducer. A cardiac artefact is then transmitted on to the flow trace while the airway is patent but this is lost when the airway obstructs. Using this indirect measure of airway patency obstruction has been detected in about half of apparently central apnoeas. ${ }^{3}$ An alternative approach is to visualise directly the upper airway during aponeic episodes and so determine its patency.

There are two likely anatomical sites for obstruction of the upper airway in apnoea, the larynx and the pharynx. Evidence exists to support obstruction at either of these sites in preterm infants with apnoea. The aim of this study was to define the site of upper airway obstruction in a group of preterm infants with severe problematical apnoea.

\section{Patients and methods}

Twelve preterm infants with severe, problematical apnoea have been studied with a median gestation of 27 weeks (range 24-31) and median birth weight $925 \mathrm{~g}$ (range 650-1630). The mean age at study was 30 days and a median postconceptional age of 30 weeks (range 27-36). Two infants were studied on more than one occasion. All but one had required initial ventilation for respiratory distress syndrome, mean duration 6.4 days (range $0-33$ ). At the time of the study recurrent apnoea was a major clinical management problem and although none were oxygen dependant when studied, supplementary oxygen was often required for apnoeic episodes. Episodes of obstruction were often suspected clinically as the apnoeic episodes were sometimes associated with hypoxia and bradycardia often requiring intervention by the nursing staff.

All infants had required treatment before the study for apnoea (table 1) and all were on methylxanthine therapy. Half of the infants required further intubation and ventilation for recurrent severe apnoea after their initial period of ventilation for lung disease. Two were found to be improved when nursed with a soft support under the neck to prevent excessive neck flexion and in three infants constant stimulation was provided by ventilating an air filled mattress.

An ultrafine fibreoptic scope (PF22Olympus) was used to visualise the upper airway of the infants studied. The scope was originally designed as an angiofibrescope for vascular surgery with an outer diameter of only $2 \cdot 2$ $\mathrm{mm}$. A similar instrument has been used previously with success as a bronchoscope in neonates. ${ }^{4}$ The scope has remote control two way angulation through $120^{\circ}$ at the tip and can be directed by the operator at the hand piece.

Table 1 Treatment for apnoed

\begin{tabular}{lc}
\hline & $N o(\%)$ of infants \\
\hline Aminophylline/theophylline & $12(100)$ \\
Intubation and ventilation & $6(50)$ \\
Nasal continuous positive airway pressure & $7(58)$ \\
Ventilated mattress & $3(25)$ \\
Neck extension & $2(17)$ \\
\hline
\end{tabular}


Images are relayed to a colour monitor by a small endoscopic camera (OTV-S2 Olympus) connected to the scope and a continuous recording made on to VHS videotape. The fine endoscope tip is introduced intranasally and advanced and directed until located at the site of interest. In this study we have concentrated on the larynx and fig 1 shows the position of the scope relative to the surrounding structures.

Simultaneous cardiorespiratory monitoring was peformed to detect spontaneously occurring apnoeas. Respiratory efforts were detected by

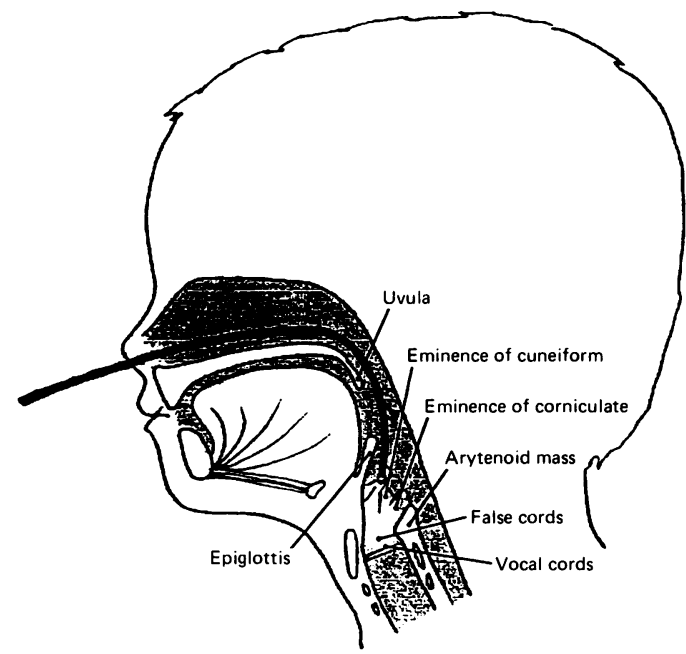

Figure 1 Position of the fibreoptic scope in relation to upper airway structures. respiratory inductive plethysmography (Respitrace system) using only the abdominal band, as previously described, in small preterm infants. ${ }^{5}$

Airflow at the nostril was recorded with a self retaining nasal thermistor. Oxygen saturation was also recorded (Ohmeda Biox 3700 pulse oximeter) together with heart rate. All monitor outputs were then recorded onto a four channel tape recorder (Racal Store 4DS) to provide a permanent recording of events.

To enable accurate timing and recognition of apnoeic events and allow direct comparison of the endoscopic view with the monitor recordings, a further modification was made. During a study the outputs of the monitors were continuously printed out on to paper by a four channel chart recorder. This print out was then filmed by a separate free standing TV camera and relayed via a mixing box (modified Sony Video Selector ITP 265A JC) to the same colour monitor displaying the laryngoscopic view (fig 2). This meant that the monitor outputs could be directly visualised alongside the picture of the upper airway and allowed the operator to observe immediately what was happening to the airway during recorded apnoeic events. When events were subsequently analysed the split screen view with both respiratory parameters and endoscopic findings were available recorded on videotape as well as the seperate permanent recording of the respiratory monitors by the four channel tape recorder.

The procedure was well tolerated by the infants and once introduced the scope appeared

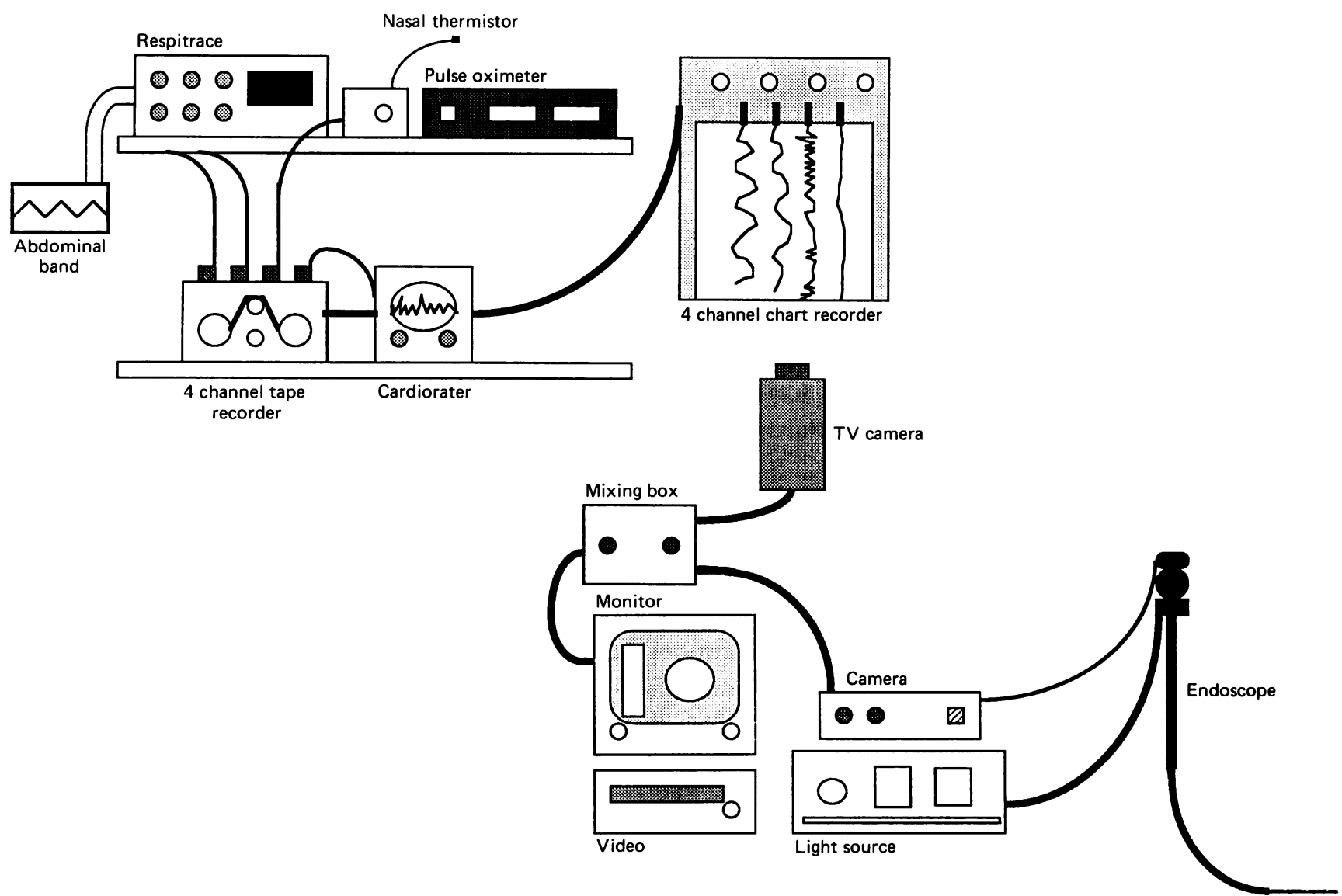

Figure 2 System to enable simultaneous visualisation of monitoring recordings and laryngoscopic view. 
Table 2 Apnoeas recorded in seven infants

\begin{tabular}{|c|c|c|c|c|c|}
\hline $\begin{array}{l}\text { Patient } \\
\text { No }\end{array}$ & Apnoea type & $\begin{array}{l}\text { Duration } \\
\text { (secs) }\end{array}$ & $\begin{array}{l}\text { Oxygen saturation } \\
\text { drop }(\%)\end{array}$ & $\begin{array}{l}\text { Lowest heart rate } \\
\text { (beats/min) }\end{array}$ & Recovery \\
\hline 1 & Obstructive* & 19 & 10 & 150 & Spontaneous \\
\hline 2 & $\begin{array}{l}\text { Mixed } \\
\text { Mixed } \\
\text { Mixed } \dagger\end{array}$ & $\begin{array}{l}50 \cdot 5 \\
45 \\
24\end{array}$ & $\begin{array}{l}36 \\
23 \\
22\end{array}$ & $\begin{array}{r}70 \\
150 \\
106\end{array}$ & $\begin{array}{l}\text { Spontaneous } \\
\text { Spontaneous } \\
\text { Intervention }\end{array}$ \\
\hline 3 & Mixed & 59 & 42 & 82 & Spontaneous \\
\hline 4 & $\begin{array}{l}\text { Mixed } \\
\text { Mixed } \\
\text { Central } \\
\text { Mixed }\end{array}$ & $\begin{array}{l}20 \\
10 \cdot 7 \\
10 \cdot 6 \\
10 \cdot 5\end{array}$ & $\begin{array}{r}18 \\
2 \\
0 \\
.0\end{array}$ & $\begin{array}{l}100 \\
171 \ddagger \\
177 \ddagger \\
170 \ddagger\end{array}$ & $\begin{array}{l}\text { Spontaneous } \\
\text { Spontaneous } \\
\text { Spontaneous } \\
\text { Spontaneous }\end{array}$ \\
\hline 5 & $\begin{array}{l}\text { Obstructive } \\
\text { Mixed }\end{array}$ & $\begin{array}{l}18 \\
39 \cdot 2\end{array}$ & $\overline{12}$ & $\begin{array}{l}97 \\
90\end{array}$ & $\begin{array}{l}\text { Spontaneous } \\
\text { Spontaneous }\end{array}$ \\
\hline 6 & Central & 30 & 32 & 90 & Spontaneous \\
\hline 7 & $\begin{array}{l}\text { Obstructive } \\
\text { Obstructive }\end{array}$ & $\begin{array}{l}43 \\
72\end{array}$ & $\begin{array}{l}61 \\
50\end{array}$ & $\begin{array}{l}78 \\
75\end{array}$ & $\begin{array}{l}\text { Intervention } \\
\text { Intervention }\end{array}$ \\
\hline
\end{tabular}

${ }^{*}$ See figure 5 , $†$ see figure 6 .

$\ddagger$ Tachycardia noted but on theophylline treatment.

to cause little discomfort or distress. Positioning of the scope was at times difficult with small movements of the baby or operator resulting in loss of the view and requiring repositioning of the scope. No increase in supplementary oxygen was necessary during the study, as judged by recorded oxygen saturations, except during apnoeic events. If the infant was on feeds these were discontinued one hour before the study and the stomach emptied to prevent aspiration.

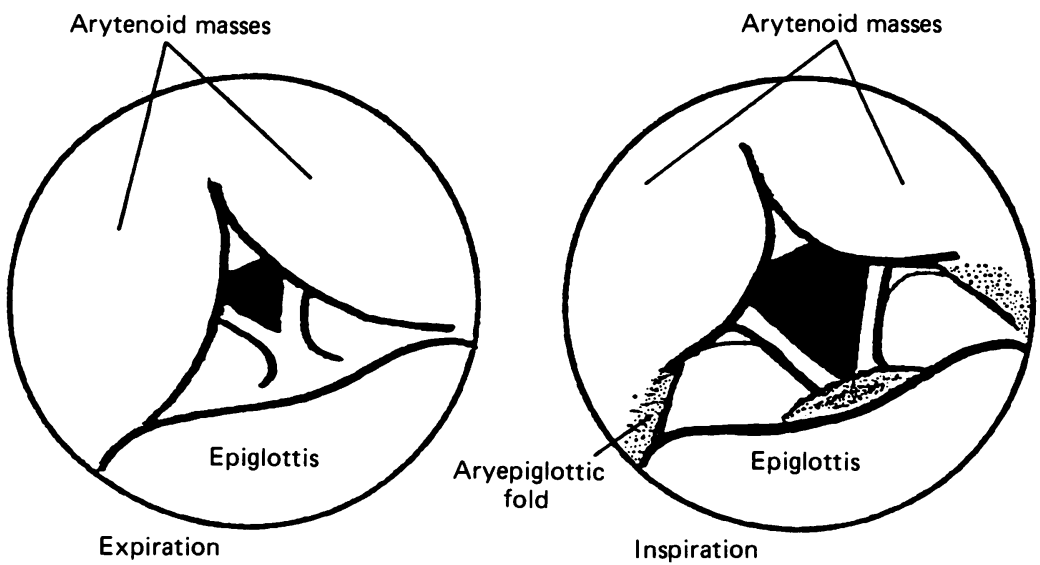

Figure 3 Laryngeal movements in tidal breathing.
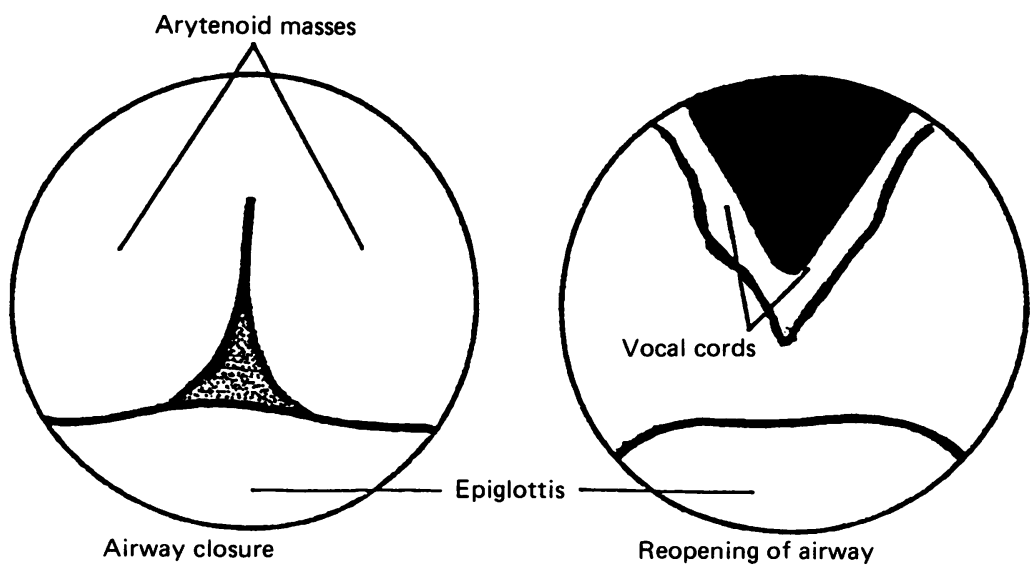

Figure 4 View of obstruction at laryngeal level during apnoea.
The infants were studied in their normal nursing position, usually prone, though some were studied supine with support to keep their neck partially extended. There were no adverse events or complications associated with the procedure.

\section{Results}

Infants were monitored for a mean time of 79 minutes (range 49-139) with simultaneous laryngoscopic visualisation for a mean time of 43 minutes (range 16-72). We were able simultaneously to record and visualise 14 apnoeic events in seven of the infants studied. The details of the apnoeas are given in table 2 . Of the apnoeas recorded eight were mixed $(57 \%)$, four obstructive ( $29 \%)$, and two central (14\%) as defined by the respiratory monitors. The mean duration was $32 \cdot 25$ seconds (range 10.5-72). During 10 of the apnoeas a drop in oxygen saturation of $10 \%$ or greater was recorded and half had associated bradycardia of less than 100 beats/minute. No apnoeas were recorded immediately on introduction or positioning of the scope. The apnoeas recorded were not uncharacteristic of the apnoeas the infants were having before the study.

\section{LARYNGOSCOPIC FINDINGS}

The majority of recording was of normal tidal breathing with cord abduction during inspiration and narrowing of the glottic aperture in expiration (fig 3). The arytenoid cartilages with their coverings and the aryepiglottic folds are prominant structures and actively mobile in the laryngeal movements of normal tidal breathing. Swallows were frequently seen with glottic closure followed by immediate reopening of the airway.

Obstruction was observed to occur at laryngeal level during the apnoeic episodes recorded (fig 4). The prominant arytenoid masses and thick aryepiglottic folds were seen to close across the airway resulting in obstruction. This was similar to that seen during swallowing but the airway patency was not restored despite, at times, respiratory efforts against the obstruc- 
tion. Figures 5 and 6 illustrate two of the apnoeic episodes recorded. The first is an obstructive apnoea with the patent laryngeal opening visualised during tidal breathing. Closure occurs at the start of the episode simultaneously with loss of airflow recorded at the nostril. Respiratory efforts continue to be made against the obstructed airway, as shown by the inductance trace, and in this example eight obstructed breaths are recorded. Airway patency is restored as airflow is again recorded with the resumption of normal respiratory effort waveforms as the obstruction is removed. The episode is associated with desaturation and a small drop in heart rate. Figure 6 is an example of a mixed apnoea with again loss of airway patency with laryngeal closure at the onset of the episode simultaneously with loss of airflow. In this case, however, there were initially no breaths against the obstruction until after about 8 seconds when respiratory efforts commence against the closed airway which remained obstructed for a further 16 seconds despite quite vigorous efforts. Again patency was restored simultaneously with the resumption of airflow. In this particular case the baby became apnoeic again shortly after and the scope withdrawn and intermittent positive pressure ventilation (IPPV) given by bag and mask. The same sequence of events was seen in the other episodes recorded including the two apparant central apnoeas with again laryngeal closure at the start of the episode with absent airflow but no respiratory efforts detected by inductance against the obstruction. Termination of the apnoea was characterised by restoration of airflow with the first respiratory effort and reopening of the laryngeal airway.

This laryngeal closure appeared to be an active process and not due to a passive collapse of the airway structures. Spontaneous recovery with reopening of the glottis and re-establishment of airway patency was seen in the majority of episodes with the scope and moni-
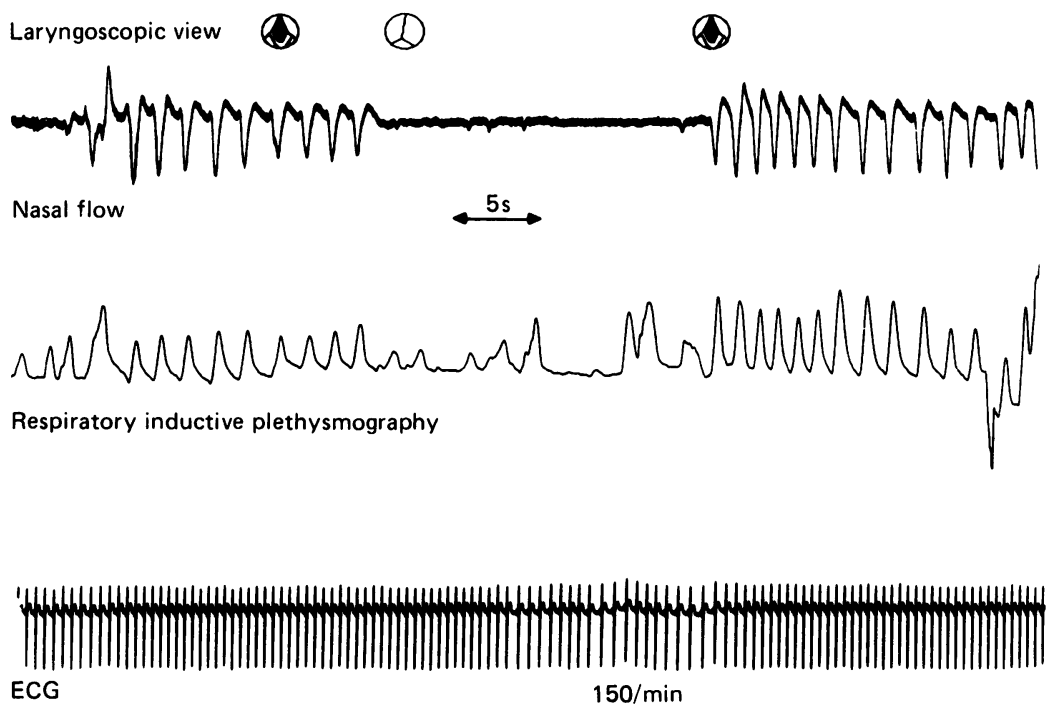

Oxygen saturation

Figure 5 Obstructive apnoea (patient 1). ECG, electrocardiography.

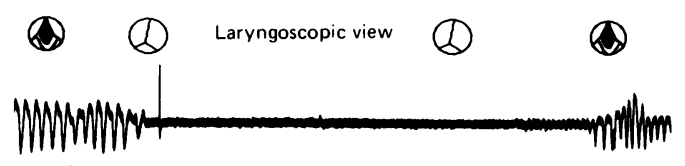

Nasal flow

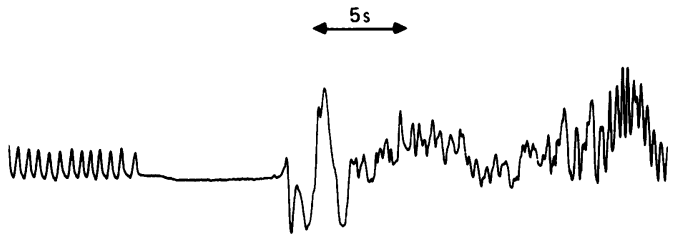

Respiratory inductive plethysmography

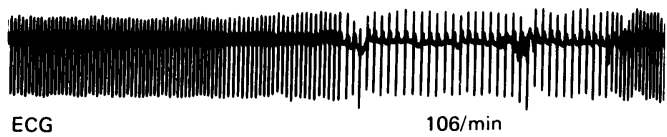

Oxygen saturation

$106 / \mathrm{min}$

Figure 6 Mixed apnoea (patient 2). ECG, electrocardiography.

toring equipment still in position. Three episodes did require active intervention to terminate, removing the scope and providing IPPV via bag and mask, but this was not unusual for those particular infants and the apnoeic episodes. When simultaneous recording and visualisation was possible we did not record any obstructive or mixed apnoea where the larynx remained patent. In six of the apnoeas, either mixed or obstructive, the accumulation of secretions before the onset of, or the clearance of secretions with recovery from, the apnoeic episode was noticed.

\section{Discussion}

The suggestion that the larynx may be the site of obstruction in preterm infants with obstructive and mixed apnoea is not new. It is a common observation in apnoeic infants that on direct laryngoscopy the cords often appear tightly adducted making intubation a difficult procedure, whereas one might expect them to lie open in a hypoxic, 'flat' infant. Steinschneider and Rabuzzi also described the resumption of respiratory efforts against such a closed glottis, as would be the situation in obstructive apnoea. ${ }^{6}$ However, it is possible that the introduction of the laryngoscope blade may itself induce glottic closure. This could also occur with our ultrafine scope yet there are a number of points to suggest that this is not the case.

Firstly, we have long periods of recording of normal tidal breathing with the scope in the same position as that during apnoeic episodes. No episodes of apnoea appeared to be precipitated by introduction or positioning of the scope. In five of the babies studied, all with recurrent apnoea, no laryngeal closure was seen for the duration of the study. We have also monitored more mature older infants with this system and have failed to see the laryngeal clo- 
sure as described during these apnoeic episodes. The apnoeas recorded in our infants were typical of the episodes experienced before study and there was no apparent increase in their frequency during the study. We also recorded each 'type' of apnoea as mixed (57\%), obstructive $(28 \%)$, and central $(14 \%)$ in the subjects and this is not far from the frequency one would expect for spontaneous apnoea particularly in a group where obstructive episodes were suspected.

In the majority of cases the apnoeas resolved spontaneously with the scope in the same position as at the onset of the episode. Research looking at laryngeal reflexes in lambs with tracheostomies showed that stimulation of various regions of the larynx with a probe, although occasionally resulting in swallows, failed to induce changes in respiratory patterns. In contrast very small volumes of hypo-osmolar fluids could produce significant respiratory depression and prolonged apnoea in the same lambs. ${ }^{7}$ This perhaps could be studied further in premature infants by spraying local anaesthetic over the laryngeal structures before scoping but this may itself be detrimental to the baby and make observations difficult to interpret. We feel confident that when performing our studies the scope itself did not precipitate any episodes of apnoea.

There is now clear evidence of a laryngeal chemoreflex in preterm infants. ${ }^{8}$ Stimulation of this reflex results in swallowing, airway obstruction, and apnoea. Many features of this airway protective mechanism are similar to spontaneous apnoeas and it has been suggested that an exaggeration of this reflex may be a cause of apnoea of prematurity. Preterm infants respond to fluid bolus volumes of as little as $0.01-0.1 \mathrm{ml}$ introduced into the pharynx. This appears to be a chemosensitive rather than simply a mechanoreceptor response due to presence of the bolus, with the response to water being greater than saline. ${ }^{9}$ Electrical stimulation of the superior laryngeal nerve produces glottic closure followed by prolonged apnoea in monkeys ${ }^{10}$ with bilateral division of these nerves abolishing the swallowing and respiratory suppression induced by this reflex. Further work in adult cats has shown continuous excitation of the thyroarytenoid muscle, with visible laryngeal adduction, follows electrical stimulation of the superior laryngeal nerve. This is accompanied by cessation of respiration and also suppression of the inspiratory activity of the laryngeal abductors, the post cricoarytenoid muscles. Similar excitation of laryngeal adductors and suppression of abductors occurs with infusion of water or sodium bicarbonate. ${ }^{11}$ Infants with malformations of the central nervous system with associated raised intracranial pressure are well documented as having apnoea and airway obstruction as a result of abductor cord paralysis. ${ }^{12}$

Bosma has given detailed attention to the anatomy of the newborn larynx describing it as 'a grossly mobile, enclosing composite of skeleton musculature and a more discretely mobile internal composite'. ${ }^{13}$ The latter is composed of the arytenoid, corniculate, aciniform, and epiglottic cartilages. He also emphasises 'the dispropor- tionate size of the arytenoid cartilages and their covering soft tissues and the prominance of the vocal, ventricular and aryepiglottic folds' resulting in a significant reduction in the size of the laryngeal lumen in comparison to that of the trachea. This agrees with our laryngoscopic findings in preterm infants with a prominant and very mobile arytenoid apparatus and the closure of these structures during airways obstruction. Swallowing produces glottic closure with cord adduction and was frequently viewed during our studies. Swallows can be produced by instillation of fluids into the oropharynx and accompany respiratory suppression as part of the laryngeal chemoreflex. The association between swallowing and apnoea has been addressed previously and has been found to occur in $76 \%$ of spontaneous prolonged apnoea. ${ }^{14}$ It often precedes apnoea and is more frequent during mixed and obstructive apnoeas. This again illustrates the intimate relationship between apnoea and airway closure at laryngeal level.

Secretions were found to be prominant at the onset of some apnoeic episodes and often their clearance was associated with recovery and reestablishment of airway patency. Oral secretions have been shown to stimulate the laryngeal chemoreflex in animals ${ }^{15}$ and it is possible that their accumulation in these infants may be involved in the aetiology of apnoeic episodes. It is certainly a common observation on our unit that babies with problematical apnoea often have copious, tenacious secretions and need frequent airway suction.

The other anatomical site proposed for upper airway obstruction in apnoea is the pharynx. This muscular tube like structure has a tendency to collapse when subject to negative intraluminal pressure. The pharyngeal muscles, particularly the genioglossus, serve to counteract this tendency but reduced tone in these muscles will predispose to obstruction at this level. This is particularly likely to be a problem in preterm infants or during rapid eye movement sleep. Certainly radiographic, electromyographic, and laryngoscopic studies indicate the oropharynx as the site of obstruction in adults and older infants with obstructive sleep apnoea. ${ }^{16} 17$ In preterm infants the use of a pharyngeal catheter monitoring pressure changes during apnoea indicated obstruction above the catheter tip high in the oropharynx in the vast majority of episodes of mixed and obstructive apnoea. ${ }^{18}$ However, the position of the catheter tip was judged by pressure changes in normal breathing and swallowing and not confirmed by laryngoscopy or radiologically. We have not focused on regions of the pharynx in this particular study as we found evidence of obstruction at the larynx. We had no incidences when we recorded mixed or obstructive apnoea where the larynx appeared patent. It is also possible that pharyngeal airway collapse may occur secondary to laryngeal obstruction as airflow through the airway ceases.

Upper airway obstruction has been visualised during central apnoea and in periods of mixed apnoea in the absence of respiratory efforts. This correlates well with the known responses 
of preterm infants to an external obstruction and other indirect indicators of airway patency. Airway obstruction may therefore be a feature of all types of preterm apnoea. This suggests that the classical division into central, mixed, and obstructive apnoea may be misleading as common underlying pathophysiological mechanisms are identified.

We thank Professor D Hull for his help with the illustrations; the Foundation for the Study of Infant Death whose financial supFoundation for the Study of Infant Death whose financial sup-
port provided the fibreoptic system; and the parents, patients, port provided the fibreoptic system; and the parents, patients,
and staff at the department of neonatal medicine and surgery, and staff at the department
Nottingham City Hospital.

1 Dransfield DA, Spitzer AR, Fox WW. Episodic airway obstruction in premature infants. Am $\mathcal{F} D$ is Child 1983;137: 441-3.

2 Milner AD, Saunders RA, Hopkin IE. Apnoea induced by airflow obstruction. Arch Dis Child 1977;52:379-82.

3 Upton CJ, Milner AD, Stokes GM. Upper airway patency during apnoea of prematurity. Annual Meeting of the British during apnoea of prematurity. Annual Meeting of the
Paediatric Association. Warwick: BPA, 1990:74.

4 Finer NN, Etches PC. Fibreoptic bronchoscopy in the neonate. Pediatr Pulmonol 1989;7:116-20.

5 Upton CJ, Milner AD, Stokes GM. Combined impedance and inductance for the detection of apnoea of prematurity. Early Hum Dev 1990;24:55-63.

6 Steinschneider A, Rabuzzi DD. Apnea and airway obstruction during feeding and sleep. Laryngoscope 1976;86: $1359-66$.
7 Harned HS, Myrade J Jr, Ferreiro J. Respiratory suppression and swallowing from introduction of fluids into the laryngeal region of the lamb. Pediatr Res 1978;12:1003-9.

8 Perkett EA, Vaughan RL. Evidence for a laryngeal chemoreflex in some human preterm infants. Acta Paediatr Scand 1982;71:969-72.

9 Davies AM, Koenig JS, Thach BT. Upper airway chemoreflex responses to saline and water in preterm infants. $\mathcal{F} \mathrm{Appl}$ Physiol 1988;64:1412-20.

10 Sutton P, Taylor EM, Lindeman RC. Prolonged apnoea in infant monkeys resulting from stimulation of superior infant monkeys resulting from stimulation of superior

11 Lucier GE, Daynes J, Sessle BJ. Laryngeal reflex regulation: peripheral and central neural analyses. Exp Neurol 1978;62: 200-13.

12 Holinger PC, Holinger LD, Reichert TJ, Holinger PH. Respiratory obstruction and apnea in infants with bilateral abductor vocal cord paralysis, meningomyelocele, hydrocephalus and Arnold-Chiari malformation. $\mathcal{F}$ Pediatr 1978; 92:368-73.

13 Bosma JF. Anatomy of the infant head. Baltimore: Johns Hopkins University Press, 1986.

14 Menon AP, Schefft GL, Thach BT. Frequency and significance of swallowing during prolonged apnoea in infants. Am Rev Respir Dis 1984;130:969-73.

15 Harding R, Johnson P, McClelland ME. Liquid-sensitive laryngeal receptors in the developing sheep, cat and monkey. F Physiol 1978;277:409-22.

16 Guillerminault G, Hill WH, Simmons FB, Dement WC Obstructive sleep apnoea: electromyographic and fibreoptic Obstructive sleep apnoea: electromyog
studies. Exp Neurol 1978;62:48-67.

17 Southall DP, Croft CB, Stebbens VA, et al. Detection of sleep associated dysfunctional pharyngeal obstruction in infants. associated dysfunctional pharyng

18 Mathew OP, Roberts JL, Thach BT. Pharyngeal airway obstruction in preterm infants during mixed and obstructive apnea. 7 Pediatr 1982;100:964-8. 